\title{
EXISTENCE OF A NON-INTERIOR PATH TO THE PERIODIC SOLUTIONS OF DIFFERENTIAL EQUATIONS
}

\section{MENGLONG SU and JIANHUA LI}

Mathematics Science College

Luoyang Normal University

Luoyang 471022

P. R. China

e-mail: mlsulynu@163.com

\begin{abstract}
In this paper, we find the periodic solutions of a class of periodicity problems via a globally convergent algorithm. Compared with the previous results in the literature, in this paper, we apply appropriate perturbations to the equality as well as inequality constraint functions and thereby are capable of choosing initial points more easily than before.
\end{abstract}

\section{Introduction}

Since Kellogg et al. (see [1]) and Smale (see [2]) proposed the notable homotopy method, this method has become a powerful tool in dealing with various nonlinear problems, for example, zeros or fixed points of maps (see [3, 4], etc. and the references therein). However, the homotopy method has seldom been touched in constrained optimization until 1988, Megiddo (see [5]) and Kojima et al. (see [6]) discovered the Karmarkar 
interior point method for linear programming was a kind of pathfollowing method. From then on, the central path following methods for mathematical programming has become an active research subject. Furthermore, it was extended to convex nonlinear programming problems recently. But all their convergence results were obtained under assumptions that the logarithmic barrier function was strictly convex and the solution set was nonempty and bounded.

In [7], for convex nonlinear programming problems, a combined homotopy interior point method was proposed. In that paper, compared with the central path following methods, the authors removed the convexity condition of the logarithmic barrier function and the boundedness and nonemptiness of the solution set.

As is well known, the periodicity problem plays a central role in the qualitative theory of differential equations for its significance in the physical sciences. Hence finding periodic solutions of ordinary differential equations is naturally an attracting topic. This paper deals with the problems of finding periodic solutions for vector ordinary differential equations of the form:

$$
x^{\prime}=f(t, x)=f(t+T, x)
$$

where $T$ is a fixed positive number. In [8], we used the homotopy interior point method to find the periodic solutions of (1.1) by introducing equality constraint functions as well as inequality constraint functions. But in [8], initial points are generally confined in the interior of $\Omega$ so that it is not easy to find an initial point for many cases, hence it is essential to enlarge the scope of choice of initial points. To this end, in this paper, we apply appropriate perturbations to the constraint functions and thereby are capable of choosing initial points more easily than before. This point may improve the computational efficiency of the algorithm greatly. 


\section{Main Results}

Set $X=\left\{x \in R^{n}: u_{i}(x) \leq 0, i=1, \ldots, m, v_{j}(x)=0, j=1, \ldots, l\right\}$, $X^{0}=\left\{x \in R^{n}: u_{i}(x)<0, i=1, \ldots, m, v_{j}(x)=0, j=1, \ldots, l\right\}, R_{+}^{m}=\left\{x \in R^{m}:\right.$ $x \geq 0\}, R_{++}^{m}=\left\{x \in R^{m}: x>0\right\}$, and $I(x)=\left\{i \in\{1, \ldots, m\}: u_{i}(x)=0\right\}$.

In [8], initial points are confined in the interior of $X$. This point may reduce the computational efficiency of predictor-corrector algorithms greatly. To enlarge the scope of choice of initial points, in this paper, we apply proper perturbation to the constraint functions $u(x), v(x)$ and introduce the parameters

$$
\gamma_{i}= \begin{cases}2 u_{i}\left(x^{(0)}\right), & u_{i}\left(x^{(0)}\right)>0, \\
1, & u_{i}\left(x^{(0)}\right)=0, i=1, \ldots, m, \quad \theta_{j}=\left\{\begin{array}{l}
1, v_{j}\left(x^{(0)}\right) \neq 0, \\
0, v_{j}\left(x^{(0)}\right)=0, \\
0, \\
u_{i}\left(x^{(0)}\right)<0,
\end{array} j=1, \ldots, l .\right.\end{cases}
$$

Then let $e_{m}=(1, \ldots, 1)^{T} \in R^{m}, \gamma=\left(\gamma_{1}, \ldots, \gamma_{m}\right)^{T} \in R^{m}, \theta=\left(\theta_{1}, \ldots, \theta_{l}\right)^{T} \in R^{l}$, $X(\lambda)=\left\{x \in R^{n}: u(x)-\lambda \gamma\left(u\left(x^{(0)}\right)+e_{m}\right) \leq 0, v(x)-\lambda \theta v\left(x^{(0)}\right)=0\right\}$, $X^{0}(\lambda)=\left\{x \in R^{n}: u(x)-\lambda \gamma\left(u\left(x^{(0)}\right)+e_{m}\right)<0, v(x)-\lambda \theta v\left(x^{(0)}\right)=0\right\}$, $I(x, \lambda)=\left\{i \in\{1, \ldots, m\}: u_{i}(x)-\lambda \gamma_{i}\left(u_{i}\left(x^{(0)}\right)+1\right)=0\right\}$.

Now we state the main result in this paper as follows:

Theorem 1. Suppose that all $u_{i}(x), i=1, \ldots, m, v_{j}(x), j=1, \ldots, l$ are $C^{3}$ functions, $\varphi_{i}\left(x, y_{i}\right), i=1, \ldots, m, \varphi_{j}\left(x, z_{j}\right), j=1, \ldots, l$ are $C^{2}$ functions, and

$\left(\mathrm{A}_{1}\right) X^{0}(\lambda)$ is nonempty and $X(\lambda)$ is bounded.

$\left(\mathrm{A}_{2}\right)$ For any $x \in X(\lambda)$, if

$$
\sum_{i \in I(x, \lambda)} \varphi_{i}\left(x, y_{i}\right)+\sum_{j=1}^{l} \psi_{j}\left(x, z_{j}\right)+\nabla v(x) \omega=0,
$$

then $y_{i}=0, \forall i \in I(x, y), z_{j}=0, j=1, \ldots, l, \omega=0$. 
$\left(\mathrm{A}_{3}\right) \varphi_{i}(x, 0)=0, i=1, \ldots, m, \psi_{j}(x, 0)=0, j=1, \ldots, l$, besides, for any $x \in X(\lambda)$, if $\|y, z, \omega\| \rightarrow \infty$, then

$$
\left\|\sum_{i \in I(x, \lambda)} \varphi_{i}\left(x, y_{i}\right)+\sum_{j=1}^{l} \psi_{j}\left(x, z_{j}\right)+\nabla v(x) \omega\right\| \rightarrow \text {. }
$$

$\left(\mathrm{A}_{4}\right)$ There exists a mapping $T\left(x, x^{(0)}\right)$ satisfying

(i) $\forall x^{(0)} \in X^{0}(\lambda), T\left(x, x^{(0)}\right)=0$ if and only if $x=x^{(0)}$.

(ii) $\forall x^{(0)} \in X^{0}(\lambda), \partial T\left(x, x^{(0)}\right) / \partial x^{(0)}$ is a matrix of full column rank.

(iii) $\forall x \in X(\lambda)$, if $T\left(x, x^{(0)}\right) \neq 0$, then

$$
T\left(x, x^{(0)}\right)+\sum_{i \in I(x, \lambda)} \varphi_{i}\left(x, y_{i}\right)+\sum_{j=1}^{l} \psi_{j}\left(x, z_{j}\right) \neq 0 .
$$

$\left(\mathrm{A}_{5}\right)$ For any $x \in X$, one has

$x(T, x) \neq x+\sum_{i=1}^{m} \varphi_{i}\left(x, y_{i}\right)+\sum_{j=1}^{l} \phi_{j}\left(x, z_{j}\right)$, for $y \in R_{+}^{m}$ with some $y_{i}>0$.

$\left(\mathrm{A}_{6}\right)$ The matrix $\nabla v(x)^{T}$ is of full row rank and $\nabla v(x)^{T} \nabla_{z}$ $\left(\sum_{j=1}^{l} \psi_{j}\left(x, z_{j}\right)\right)$ is nonsingular.

Then for almost every $P^{(0)}=\left(x^{(0)}, y^{(0)}, z^{(0)}\right) \in R^{n} \times R_{++}^{m} \times R^{l}$, and $\lambda \in(0,1]$, there exists a $C^{1}$ curve $(P(s), \lambda(s))$ of dimension 1 of the homotopy 
$H(P, \lambda)=\left(\begin{array}{c}(1-\lambda)(x-x(T, x))+\sum_{i=1}^{m} \varphi_{i}\left(x,(1-\lambda) y_{i}\right)+(1-\lambda) \lambda \nabla v(x) z \\ +\sum_{j=1}^{l} \psi_{j}\left(x, z_{j}\right)+\lambda T\left(x, x^{(0)}\right)+\lambda(1-\lambda) \alpha \\ v(x)-\lambda \theta v\left(x^{(0)}\right) \\ \operatorname{diag}(y)\left(u(x)-\lambda \operatorname{diag}(\gamma)\left(u\left(x^{(0)}\right)+e_{m}\right)\right)-\lambda \operatorname{diag}\left(y^{(0)}\right)\left(u\left(x^{(0)}\right)\right. \\ \left.-\operatorname{diag}(\gamma)\left(u\left(x^{(0)}\right)+e_{m}\right)\right)\end{array}\right)=0$

such that the limit set $T \subset X^{0} \times R_{+}^{m} \times\{0\}$ is nonempty, in particular, $x\left(t, x^{*}\right)$ is a T-periodic solution of (1.1).

It should be pointed out that the results in this paper also extend the results in [9] from inequality cases to equality cases.

The zero-point set of $H$ is

$$
H^{-1}(0)=\left\{(P, \lambda) \in X(\lambda) \times R_{+}^{m} \times R^{l} \times(0,1]: H(P, \lambda)=0\right\} .
$$

The inverse image theorem tells us that, if 0 is a regular value of the map $H_{P^{(0)}}$, then $H_{P^{(0)}}^{-1}(0)$ consists of some smooth curves. And the regularity of $H_{P^{(0)}}$ can be obtained by the following lemma:

Lemma 2.1 (Parameterized Sard's Theorem). Let $V \subset R^{n}, U \subset R^{m}$ be open sets, and $\Phi: V \times U \rightarrow R^{k}$ a $C^{r} \operatorname{map}$, where $r>\max \{0, m-k\}$. If $0 \in R^{k}$ is a regular value of $\Phi$, then for almost all $a \in V, 0$ is a regular value of $\Phi_{a} \equiv \Phi(a, \cdot)$.

The method presented in this paper may also give a effective way for other periodicity problems, for example, Duffing equation or Liénard equation

$$
x^{\prime}=y-F(x),
$$




$$
y^{\prime}=-x,
$$

where $\quad F(x)=\int_{0}^{x} f(\xi) d \xi$. Set $\quad X=(x, y)^{T}, f(t, X)=(y-F(x),-x)^{T}$. Then (2.2) and (2.3) becomes (1.1).

Proof of Theorem 1. When $x^{(0)}$ and $\alpha$ are considered as variables, we denote $H(P, \lambda)$ by $\bar{H}\left(P, x^{(0)}, \alpha, \lambda\right)$. Let the Jacobian matrix of $\bar{H}\left(P, x^{(0)}, \alpha, \lambda\right)$ be denoted by $D \bar{H}\left(P, x^{(0)}, \alpha, \lambda\right)$, for any $\lambda \in(0,1]$,

$$
\begin{array}{lc}
\frac{\partial \bar{H}\left(P, x^{(0)}, \alpha, \lambda\right)}{\partial\left(x^{(0)}, \alpha, \lambda\right)}= & \\
\left(\begin{array}{ccc}
\lambda \frac{\partial T\left(x, x^{(0)}\right)}{\partial x^{(0)}} & \lambda(1-\lambda) I & (1-\lambda) \nabla_{y}\left(\sum_{i=1}^{m} \varphi_{i}\left(x,(1-\lambda) y_{i}\right)\right) \\
-\lambda \theta \nabla v\left(x^{(0)}\right)^{T} & 0 & 0 \\
-\lambda \gamma \operatorname{diag}(y) \nabla u\left(x^{(0)}\right)^{T} & 0 & \operatorname{diag}\left(u(x)-\operatorname{diag}(\gamma)\left(u\left(x^{(0)}\right)+e_{m}\right)\right)
\end{array}\right) .
\end{array}
$$

Since $\nabla v(x)^{T}$ is a matrix of full row rank, $\partial \bar{H}\left(P, x^{(0)}, \alpha, \lambda\right) / \partial\left(x^{(0)}, \alpha, y\right)$ is of full row rank. Therefore $D \bar{H}\left(P, x^{(0)}, \alpha, \lambda\right)$ is also of full row rank, and 0 is a regular value of $\bar{H}\left(P, x^{(0)}, \alpha, \lambda\right)$. By Lemma 2.1, for almost all $\left(x^{(0)}\right) \in X^{0}(1), 0$ is a regular value of map $H: R^{n} \times R_{+}^{m} \times R^{l} \times(0,1]$ $\rightarrow R^{n+m+p}$. By the inverse image theorem, $H^{-1}(0)$ consists of some smooth curves. Since $H\left(P^{(0)}, 1\right)=0$, then a $C^{1}$ curve $(P(s), \lambda(s))$ of dimension 1 , denoted by $\Gamma_{P^{(0)}}$, is starting from $\left(P^{(0)}, 1\right)$.

By the classification theorem of one-dimensional smooth manifold, $\Gamma_{P^{(0)}}$ is diffeomorphic to a unit circle or the unit interval $(0,1]$. Since the matrix 


$$
\frac{\partial H\left(P^{(0)}, 1\right)}{\partial P}=\left(\begin{array}{ccc}
\frac{\partial T\left(x, x^{(0)}\right)}{\partial x} & 0 & \nabla_{z}\left(\sum_{j=1}^{l} \psi_{j}\left(x, z_{j}\right)\right. \\
\nabla v\left(x^{(0)}\right)^{T} & 0 & 0 \\
\operatorname{diag}\left(y^{(0)}\right) \nabla u\left(x^{(0)}\right)^{T} & \operatorname{diag}\left(u\left(x^{(0)}\right)\right) & 0
\end{array}\right)
$$

is nonsingular, so $\Gamma_{P^{(0)}}$ is diffeomorphic to a unit interval.

Let $\left(P^{*}, \lambda^{*}\right)$ be a limit point of $\Gamma_{P^{(0)}}$, then the following cases may occur:

(a) $\left(P^{*}, \lambda^{*}\right)=\left(x^{*}, y^{*}, z^{*}, \lambda^{*}\right) \in X \times R_{+}^{m} \times R^{l} \times\{0\}$,

(b) $\left(P^{*}, \lambda^{*}\right)=\left(x^{*}, y^{*}, z^{*}, \lambda^{*}\right) \in X^{0}(1) \times R_{++}^{m} \times R^{l} \times\{1\}$,

(c) $\left(P^{*}, \lambda^{*}\right)=\left(x^{*}, y^{*}, z^{*}, \lambda^{*}\right) \in \partial\left(X\left(\lambda^{*}\right) \times R_{+}^{m} \times R^{l}\right) \times(0,1]$.

By Lemma 2.1, the equation $H\left(P^{(0)}, 1\right)=0$ has a unique solution $\left(P^{(0)}, 1\right)$ in $X^{0}(1) \times R_{++}^{m} \times R^{l} \times\{1\}$, so case (b) won't occur.

It is easy to show that the projection of the smooth curve $\Gamma_{P^{(0)}}$ on the $z$-plane is bounded.

If case (c) holds, then there exists a sequence of points $\left\{\left(P^{(k)}, \lambda_{k}\right)\right\} \subset \Gamma_{P^{(0)}}$ such that $\left\|\left(P^{(k)}, \lambda_{k}\right)\right\| \rightarrow \infty$. Since $X(\lambda)$ and $(0,1]$ are bounded, hence there exists a subsequence of points (denoted also by $\left.\left\{\left(P^{(k)}, \lambda_{k}\right)\right\}\right)$ such that $x^{(k)} \rightarrow x^{*},\left\|y^{(k)}\right\| \rightarrow \infty, z^{(k)} \rightarrow z^{*}$, and $\lambda_{k} \rightarrow \lambda^{*}$ as $k \rightarrow \infty$. From the third equation of (2.1), we have

$$
\begin{aligned}
u\left(x^{(k)}\right) & -\lambda_{k} \operatorname{diag}(\gamma)\left(u\left(x^{(0)}\right)+e_{m}\right) \\
\quad= & -\lambda_{k}\left(\operatorname{diag}\left(u^{(k)}\right)\right)^{-1} \operatorname{diag}\left(y^{(0)}\right)\left(u\left(x^{(0)}\right)-\operatorname{diag}(\gamma)\left(u\left(x^{(0)}\right)+e_{m}\right)\right) .
\end{aligned}
$$


When $\lambda^{*}>0$, the active index set

$$
I\left(x^{*}, \lambda^{*}\right)=\left\{i \in\{1, \ldots, m\}: \lim _{k \rightarrow \infty} y_{i}^{(k)}=\infty\right\} .
$$

When $\lambda^{*}=0$, the index set

$$
I_{0}\left(x^{*}, 0\right)=\left\{i \in\{1, \ldots, m\}: \lim _{k \rightarrow \infty} y_{i}^{(k)}=\infty\right\} \subset I\left(x^{*}, 0\right) .
$$

From the homotopy equation (2.1), we have

$$
\begin{aligned}
\sum_{i \in I\left(x^{*}, \lambda^{*}\right)} \varphi_{i}\left(x^{(k)},\left(1-\lambda_{k}\right) y_{i}^{(k)}+\sum_{j=1}^{l} \phi_{j}\left(x^{(k)}, z_{j}^{(k)}\right)+\lambda_{k} T\left(x^{(k)}, x^{(0)}\right)\right. \\
=-\left(1-\lambda_{k}\right)\left(x^{(k)}-x\left(T, x^{(k)}\right)+\lambda_{k}\left(\alpha+\nabla v\left(x^{(k)}\right) z^{(k)}\right)\right) \\
\quad-\sum_{i \notin I\left(x^{*}, \lambda^{*}\right)} \varphi_{i}\left(x^{(k)},\left(1-\lambda_{k}\right) y_{i}^{(k)}\right) .
\end{aligned}
$$

By the fact that $y_{i}^{(k)}$ is bounded for $i \notin I\left(x^{*}, \lambda^{*}\right)$ and the fact that $X\left(\lambda^{*}\right)$ is bounded, when $k \rightarrow \infty$, from (2.4), let $y_{i}^{*}=\lim _{k \rightarrow \infty}\left(1-\lambda_{k}\right) y_{i}^{(k)}$, we have

$$
\sum_{i \in I\left(x^{*}, 1\right)} \varphi_{i}\left(x^{*}, y_{i}^{*}\right)+\sum_{j=1}^{l} \psi_{j}\left(x^{*}, z_{j}^{*}\right)+T\left(x^{*}, x^{(0)}\right)=0
$$

which contradicts assumption $\left(\mathrm{A}_{4}\right)$.

If $0 \leq \lambda^{*}<1$, from (2.4), when $k \rightarrow \infty$, since $X\left(\lambda^{*}\right)$ and $y_{i}^{(k)}$, $i \notin I\left(x^{*}, \lambda^{*}\right)$ are bounded, the right-hand side of (2.4) is bounded. At the same time, the left-hand side of (2.4) is infinite. This point results in a contradiction. 
By the above discussion, we obtain that case (a) is the only possible case. Therefore $\left(x^{*}, y^{*}\right)$ is a solution of the system

$$
\begin{aligned}
& x-x(T, x)+\sum_{i=1}^{m} \varphi_{i}\left(x, y_{i}\right)+\sum_{j=1}^{l} \psi_{j}\left(x, z_{j}\right)=0, \\
& v(x)=0, \\
& \operatorname{diag}(y) u(x)=0, u(x) \leq 0, y \geq 0 .
\end{aligned}
$$

Then it is easy to show that $x\left(t, x^{*}\right)$ is a $T$-periodic solution of (1.1).

\section{Acknowledgements}

This work was supported by National Nature Science Foundation of China (No. U1304103), Innovation Scientists Technicians Troop Construction Projects of Henan Province (No. C20150027), and

Innovation Scientists Technicians Troop Construction Projects of Luoyang Normal University (No.2014-CXTD-001).

\section{References}

[1] R. B. Kellogg, T. Y. Li and J. A. Yorke, A constructive proof of the Brouwer fixedpoint theorem and computational results, SIAM J. Numer. Anal. 13 (1976), 473-483.

[2] S. Smale, A convergent process of price adjustment and global Newton method, J. Math. Econ. 3 (1976), 1-14.

[3] S. N. Chow, J. Mallet-Paret and J. A. Yorke, Finding zeros of maps: Homotopy methods that are constructive with probability one, Math. Comput. 32 (1978), 887-899.

[4] C. B. Garcia and W. I. Zangwill, An approach to homotopy and degree theory, Math. Oper. Res. 4 (1979), 390-405.

[5] N. Megiddo, Pathways to the optimal set in linear programming, in Progress in Mathematical Programming, Interior Point and Related Methods (N. Megiddo, ed.), Springer, New York, 1988, pp. 131-158.

[6] M. Kojima, S. Mizuno and A. Yoshise, A primal-dual interior point algorithm for linear programming, Interior Point and Related Methods (N. Megiddo, ed.), Springer, New York, 1988, pp. 29-47. 
[7] Z. H. Lin, B. Yu and G. C. Feng, A combined homotopy interior point method for convex nonlinear programming, Appl. Math. Comput. 84 (1997), 193-211.

[8] M. L. Su, Existence of the periodic solutions of a class of periodicity problem of differential equations and related algorithms, Advances in Differential Equations and Control Processes 15(2) (2015), 83-92.

[9] M. L. Su and S. Y. Nie, A non-interior path following method for solving the periodicity problem of a class of differential equations, Advances in Differential Equations and Control Processes 15(1) (2015), 45-51. 